\title{
AVALIAÇÃO BIOMÉTRICA DO OVIDUTO DE MARRECAS DE ROUEN
}

\author{
FRANZO, Vanessa Sobue ${ }^{1}$ \\ MORAES, Carime ${ }^{2}$ \\ VULCANI, Valcinir Aloisio Scalla ${ }^{3}$ \\ GRADELA, Adriana ${ }^{4}$ \\ ARTONI, Silvana Martinez Baraldi ${ }^{5}$
}

\begin{abstract}
RESUMO: Foram estudados parâmetros biométricos do oviduto de 10 marrecas de Rouen (Anas playtrynchus) adultas em fase reprodutiva. As aves foram eutanasiadas, evisceradas e dissecadas e o oviduto foi retirado e pesado em uma balança de precisão e logo depois, estendido em uma superfície plana e sem sofrer estiramento, foi medido com uma fita métrica. Os segmentos anatômicos do oviduto foram separados e medidos individualmente. Posteriormente, contou-se as pregas do magno e istmo. Os dados foram submetidos a teste de T de Student com o nível de significância $\mathrm{p} \leq 0,05$. Os resultados foram: peso do oviduto $(47,78 \pm 3,47 \mathrm{gr}) ; 7,80 \pm$ $0,40 \mathrm{~cm}$ de infundíbulo; $29,09 \pm 4,34 \mathrm{~cm}$ de magno; $10,11 \pm 0,34 \mathrm{~cm}$; de istmo; $6,45 \pm 2,26 \mathrm{~cm}$ de útero; $8,01 \pm$ $2,71 \mathrm{~cm}$ de vagina; comprimento médio do oviduto $(56,00 \pm 3,60 \mathrm{~cm}$ ); número médio de pregas do magno (de $15,00 \pm 2,30)$; número médio de pregas do istmo $(13,00 \pm 2,44)$ e, finalmente, vagina com média de oito anéis na porção inicial. Conclui-se que o oviduto é mais leve do que os de peruas e galinhas domésticas, porém, seu comprimento é maior do que o de galinhas. O magno é a maior porção do oviduto de marrecas de Rouen. O istmo e o útero possuem comprimentos maiores do que em galinhas.
\end{abstract}

Palavras-chave: Anatomia. Reprodução. Aves. Preservação. Sistema reprodutor.

\section{BIOMETRIC PARAMETERS OF THE OVIDUCT OF THE ROUEN DUCK}

SUMMARY: Biometric parameters were studied in the oviduct of 10 of Rouen ducks (Anas playtrynchus) adults in the reproductive phase. The birds were euthanized, eviscered and dissected and their oviduct was retired and was weighed on electronic precision scale and the viscera was placed on a horizontal plane surface and was measured with a tape measure and the anatomical segments of the oviduct were separated and measured individually. Then, the folds of the magnum and the isthmus were counted. The statistical analysis utilized was Student's -test with a significance level $\mathrm{p} \leq 0.05$. The results showed that the duck possess: weight of the oviduct $(47.78 \pm 3.47 \mathrm{gr}) ; 7.80 \pm 0.40 \mathrm{~cm}$ of infundibulum; $29.09 \pm 4.34 \mathrm{~cm}$ magnum; $10.11 \pm 0.34 \mathrm{~cm}$ of the isthmus; $6.45 \pm 2.26 \mathrm{~cm}$ of the isthmus; $8.01 \pm 2.71 \mathrm{~cm}$ of the vagina, the average length of oviduct $(56.00 \pm$ $3.60 \mathrm{~cm})$, average number of folds of the magnum $(15.00 \pm 2.30)$, average number of folds of the isthmus (13.00 \pm 2.44 ) and finally, vagina with eight rings in the initial portion. Conclude that the oviduct is lighter than

\footnotetext{
${ }^{1}$ Dra. em Medicina Veterinária (Patologia Animal) pela FCAV-UNESP. Docente adjunto da UFMT, campus Cuiabá, Departamento de Zootecnia e Extensão Rural.

${ }^{2}$ Doutoranda. Departamento de Morfologia e Fisiologia Animal. Faculdade de Ciências Agrárias e Veterinárias de Jaboticabal (FCAVJ).Universidade Estadual Paulista (UNESP), Jaboticabal.

3 Dr. em Cirurgia Experimental pela FCAV, UNESP. Docente adjunto do campus Avançado de Jataí, Universidade Federal de Goiás, Jataí.

${ }^{4}$ Da. em Produção Animal pela FCAV, UNESP. Docente adjunto I. Universidade Federal do Vale do São Francisco, Campus de Ciências Agrárias. Petrolina, Pernambuco.

${ }^{5}$ Livre docente do departamento de morfologia e fisiologia animal da FCAV-UNESP.
} 
domestic chickens and turkeys, but its length is greater than chickens. The magnum is the largest portion of the oviduct of ducks. The isthmus and uterus are longest than chickens.

Keywords: Anatomy. Reproduction. Bird. Preservation. Reproductive system.

\section{INTRODUÇÃO}

Anatidae é uma família de aves que tem como representantes os cisnes, os marrecos e os patos (SICK, 2001). São facilmente adaptáveis em cativeiro e muito resistentes às doenças aviárias mais comuns que acometem galinhas (FABICHACK, 2000). Os ovos são muito consumidos em países europeus e, no Brasil é bastante relevante na região Sul, em que são utilizados como alimento e empregados na preparação de vacinas em empresas comerciais (FABICHACK, 2000).

Dyce et al. (2004) relataram que os órgãos reprodutores da fêmea são constituídos pelo ovário e o oviduto, os quais são bilateralmente simétricos na vida embrionária. Mas Sultana et al. (2003) e Dyce et al. (2004) observaram que, em aves domésticas, somente o ovário e o oviduto esquerdo são funcionais. De acordo com Dyce et al. (2004), o oviduto conduz o ovo fertilizado para a cloaca, assim como, adiciona grandes quantidades de nutrientes ao ovo, além do envolvimento com membranas e casca para a proteção do embrião. King et al. (2002) observaram que, o oviduto das aves possuem a capacidade de armazenar o espermatozóide por períodos prolongados, cujo tempo de permanência depende de fatores como a espécie e a idade da ave e o estado reprodutivo dela.

De acordo com Sultana et al. (2003) e Dyce et al. (2004) o oviduto pode ser dividido, anatomicamente, em cinco regiões distintas e com funções fisiológicas específicas: infundíbulo, magno (região secretora de albúmen), istmo, útero (glândula da casca) e vagina.

O objetivo do presente estudo é a avaliação biométrica do oviduto de marrecas de Rouen (Anas platyrynchus) visando fornecer dados biológicos factíveis para ampliação de conhecimentos relacionados à sua biologia reprodutiva, em aspectos práticos, como a criação comercial, a preservação e o controle biológico da espécie avícola em estudo.

\section{MATERIAL E MÉTODO}

Utilizou-se 10 marrecas de Rouen (Anas platyrynchus) adultas, com peso corpóreo de, aproximadamente, 2650 gramas, em fase reprodutiva proveniente do Departamento de Patologia Animal da Universidade Estadual Paulista, Faculdade de Ciências Agrárias e Veterinárias (Unesp, FCAV), campus Jaboticabal. As aves foram anestesiadas com 
Zolazepam na dose $10 \mathrm{mg} / \mathrm{Kg}$ intramuscular no músculo peitoral superficial e, em seguida, utilizou-se cloreto de potássio na dose de $2 \mathrm{mg} / \mathrm{Kg}$ intravenoso de peso vivo para a eutanásia do animal. Depois da abertura das cavidades torácica e abdominal, ocorreu o rebatimento do esterno e retirou-se as vísceras abdominais para a coleta dos ovidutos.

O oviduto foi pesado com o auxílio de uma balança de precisão e medido com o auxílio de uma fita métrica milimetrada. Além disso, seus segmentos anatômicos (infundíbulo, magno, istmo, útero e vagina) foram medidos utilizando-se uma fita métrica milimetrada. Para procedimentos de medida, o oviduto foi colocado, sem sofrer estiramento sobre uma superfície plana. Posteriormente contou-se o número de pregas do magno e istmo.

Os dados foram analisados estatisticamente pelo teste $\mathrm{T}$ de Student com o nível de significância $\mathrm{p} \leq 0,05$ e os termos utilizados neste trabalho estão de acordo com a Nomina anatomica avium (BAUMEL, 1993).

O peso médio do oviduto da marreca de Rouen, em atividade reprodutiva, foi de 47,78 $\pm 3,47$ gramas. O peso do oviduto de galinhas de postura e de quatro linhagens de peruas foram reportados por Dalrymple et al. (1968) e Verma e Cherms (1964) cujos pesos médios variaram de 265 a 279 gramas.

O comprimento total do oviduto em marrecas de Rouen em período reprodutivo foi de $56 \mathrm{~cm}$, em média, maior do que o de galinhas domésticas foi de $50 \mathrm{~cm}$ (SISSON; GROSSMAN, 1996) e menor do que o de peruas, $85 \mathrm{~cm}$ (VERNA; CHERMS, 1964). O comprimento do oviduto está relacionado ao ciclo reprodutivo do animal, pois na fase de inatividade sexual, o oviduto apresenta-se pequeno e menos volumosos, enquanto que, na puberdade e na fase de postura ocorre um aumento de tamanho de todo o oviduto (NIKEL et al., 1977).

Ao analisar as partes do oviduto notou-se que eles obtiveram um comprimento médio de: infundíbulo com 7,80 \pm 0,40 cm; magno, 29,09 \pm 4,34 cm; istmo apresentando $10,11 \pm$ $0,34 \mathrm{~cm}$; útero medindo $6,45 \pm 2,26 \mathrm{~cm}$ e, finalmente a vagina com $8,01 \pm 2,71 \mathrm{~cm}$. Além disso, verificou-se que o número médio de pregas do magno foi de 15,00 \pm 2,30 e o número de pregas do istmo foi de $13,00 \pm 2,44$. Ainda, em relação à vagina, observou-se que há, em média, 8 anéis na porção inicial.

O infundíbulo secreta material protéico depositado ao redor da gema e contribui para a resistência da membrana pré-vitelina de acordo com Sisson e Grossman (1986) e Hafez (1988). Em marrecas de Rouen o infundíbulo $(7,80 \pm 0,40 \mathrm{~cm})$ é maior do que o de peruas (VERNA; CHERMS, 1964) e de codornas (MORAES et al., 2007). 
O magno é a porção mais longa do oviduto de marrecas de Rouen, com 29,09 $\pm 4,34 \mathrm{~cm}$ de comprimento, concordando com Gilbert (1972), ao estudar o sistema reprodutor de aves e também, com King (1975). O comprimento do oviduto de marrecas de Rouen é similar ao da galinha doméstica (30 cm) conforme citado por Dyce et al., (2004), porém é menor do que o de peruas Broad Breasted Bronze que possuem o magno com maior comprimento $(38,4 \mathrm{~cm})$ como relatado por Verna e Cherms (1964). Moraes et al. (2007) ao estudarem codornas (Nothura maculosa) notaram o magno com menor comprimento $(15,2 \mathrm{~cm})$ do que o de marrecas de Rouen, igualmente como em patas $(20 \mathrm{~cm})$ como observado por Das et al., (1965). Além disso, o magno de marrecas de Rouen possui paredes mais espessas devido à presença de glândulas em seu interior (JUNQUEIRA; CARNEIRO, 2004) cuja função é a formação do albúmen do ovo (DYCE et al., 2004). As 15 pregas, em média, presentes no magno são mais altas e mais espessas da mucosa do oviduto, enquanto os últimos centímetros do magno apresentam pregas reduzidas, assim como ocorre em Nothura maculosa (MORAES et al., 2007).

O istmo de marrecas quando comparado com o de galinhas domésticas é maior, apresenta um comprimento médio de $10,11 \pm 0,34 \mathrm{~cm}$, enquanto que, o comprimento dessa estrutura anatômica em galinhas é de $8 \mathrm{~cm}$ (DYCE et al, 2004), porém é menor do que o de peruas, que apresenta 16,4 cm, aproximadamente como estudado por Verna e Cherma (1964). Tal segmento é responsável pela formação da membrana da casca e uma pequena quantidade de albúmen (SISSON; GROSSMAN, 1996). De acordo com Dyce et al. (2004), a membrana interna da casca é sintetizada assim que a gema penetra no istmo e a membrana externa quando o ovo começa a se deslizar neste compartimento.

O útero é uma região muscular expandida (SISSON; GROSSMAN, 1996; DYCE et al., 2004) e em marrecas mediu cerca de $6,45 \pm 2,26 \mathrm{~cm}$, enquanto que, a galinha em postura apresenta um comprimento médio de $8 \mathrm{~cm}$ (DYCE et al., 2004), assim como patas $(9 \mathrm{~cm})$ como observado por Das et al., (1965). No útero ocorre a formação da casca do ovo no período de 20 horas e 40 minutos (KING, 1975).

A vagina é um estreito tubo muscular (FUJII; TAMURA, 1963) sendo que, essa estrutura anatômica em marrecas de Rouen é constituída, em média, por oito anéis musculomembranosos sendo relacionados com a expulsão do ovo já formado em partes anteriores do oviduto (SISSON e GROSSMAN, 1996). King (1975) e Dyce et al, (2004) observaram ausência desses anéis em galiniformes (KING, 1975; DYCE, 1996). O comprimento de $8,01 \pm 2,71 \mathrm{~cm}$ de marrecas se assemelha ao encontrado por Verna e Cherms ao estudarem peruas $(10,6 \mathrm{~cm})$, porém é maior do que o de patas reportado por Das et al. (1965). 


\section{CONCLUSÃO}

Conclui-se, anatomicamente, que as porções do oviduto de marrecas de Rouen são similares ao de galinhas domésticas. Estas aves possuem o oviduto é mais leve do que os de peruas e galinhas domésticas, porém, seu comprimento é maior do que o de galinhas. $\mathrm{O}$ magno é a maior porção do oviduto de marrecas de Rouen. O istmo e o útero possuem comprimentos maiores do que em galinhas.

\section{REFERÊNCIAS}

BAUMEL, J. J. Handbook of avian anatomy: Nomina anatomica avium. 2.ed. Cambridge: Nuttal Ornithological Club, 1993. 779 p.

DAS, L. N.; BISWAL, G. Microanatomy of the reproductive tract of domestic duck ( Anas boscas). The Indian Veterinary Journal, Orissa, v. 45, p. 1003-1009, 1968.

DALRYMPLE, J. R.; MACPHERSON, J. W., FRIARS, G. W. The reproductive tract of the turkey hen (a biometrical study). Canadian Journal Compedium Medicine. v. 32, p. 435, 1968.

DYCE, K. M.; SACK, W. O.; WENSING, C. J. G. Tratado de anatomia veterinária. 2. ed. Elsevier, 2004.872 p.

FABICHACK, I. Criação de Patos e Marrecos. São Paulo: Nobel, 2000. p. 01-72.

FUJII, S.; TAMURA, T. Location of sperms in the oviduct of the domestic fowl with special reference to storage of sperms in the vaginal gland. Journal of Fish Animal Husb, Hiroshima, v. 5, p.145-163, 1963.

GILBERT, A. B. The activity of the ovary in relation to egg production. In: FREEMAN, B. M.; LAKE, P. E. Egg production and formation. Edinburg: Poultry Science, 1972. v. 4, cap.21, p. 488-515.

HAFEZ, E. S. E. Reprodução animal. 4 ed. São Paulo: Manole. 1988. 720 p.

JUNQUEIRA, L. C.; CARNEIRO, J. Histologia básica. 10. ed. Rio de Janeiro: Guanabara Koogan, 2004. 540 p.

KING, A. S. Introdução às aves. IN: SISSON, S.; GROSSMAN, J. D. Anatomia dos animais domésticos. Rio de Janeiro: Guanabara Koogan, 1986. v. 2, cap.59, p. 1677-1679.

KING, L. M.et al. Segregation of spermatozoa within sperm storage tubules of fowl and turkey hens. Reproduction, Cambridge, v.123, n.1, p.79-86, 2002.

MORAES, C.et al. Morfologia e morfometria do oviduto de codornas Nothura maculosa. Ciência Rural, Santa Maria, v. 37, n. 1, p. 146-152, 2007. 
NICKEL, R.; SCHUMMER, A.; SEIFERLE, E. Anatomy of the domestic birds. Berlim: Verlag Paul Parey, p. 41-50, 1977.

SICK, H. Ornitologia Brasileira. Família Falconidae. $3^{\mathrm{a}}$ impressão. Rio de Janeiro: Nova Fronteira, 2001. parte 2, cap. 10, p. 243-269,

SISSON, S.; GROSSMAN, J. D. Anatomia dos animais domésticos. 5 ed. Rio de Janeiro:Guanabara Koogan,1986. 2000 p.

SULTANA, F. et al. The peri-albumen layer: a novel structure in the envelopes of avian egg. Journal of Anatomy, Cambridge, v. 203, n. 1, p. 115-122, 2003.

VERMA, O. P.; CHERMS, F. L. Observations on the oviduct of the turkeys. Avian Diseases, Vennete Square, v. 8, p. 19-26, 1964. 